\title{
$M R$ venography in idiopathic intracranial hypertension: unappreciated and misunderstood
}

\author{
J N P Higgins, J H Gillard, B K Owler, K Harkness, J D Pickard
}

J Neurol Neurosurg Psychiatry 2004;75:621-625. doi: 10.1136/jnnp.2003.021006

See end of article for authors' affiliations

Correspondence to: Dr Nicholas Higgins, Department of Radiology, Box 219, Addenbrooke's Hospital, Hills Road, Cambridge CB2 2QQ, UK; nick.higgins@ addenbrookes.nhs.uk

Received 18 June 2003 In revised form 8 August 2003

Accepted 9 August 2003
Background: Venous sinus disease must be excluded before diagnosing idiopathic intracranial hypertension but is found only rarely in typical cases. Magnetic resonance venography (MRV) is the technique of choice for investigating this, and provides images that are diagnostic and easy to interpret. However, recent work using more invasive techniques has documented pressure gradients and stenoses in the lateral venous sinuses in many cases of idiopathic intracranial hypertension.

Objective: To examine the reason for this discrepancy and to establish whether there are characteristic appearances on MRV in idiopathic intracranial hypertension that are routinely overlooked in clinical practice.

Methods: MRVs from 20 patients with idiopathic intracranial hypertension were reviewed, unblinded, by two neuroradiologists, and their appearances rated for focal narrowings and signal gaps. A control group of 40 asymptomatic volunteers, matched for age and sex with the patient group, was recruited prospectively for MRV, and their scans rated in the same way.

Results: The lateral sinuses presented a range of appearances with quite different distributions in the two groups $(p<0.001)$. Bilateral lateral sinus flow gaps were seen in 13 of 20 patients with idiopathic intracranial hypertension and in none of 40 controls.

Conclusions: A historical failure to use normal healthy controls to establish the boundaries between imaging artefact, normal anatomical variant, and disease means that the pathological significance of the different appearances of the lateral sinuses on MRV has not so far been appreciated.
$\mathrm{T}$ he aetiology of idiopathic intracranial hypertension is unknown, but similarities with cerebral venous sinus thrombosis are recognised. Sagittal sinus thrombosis, in particular, must be excluded before idiopathic intracranial hypertension can be diagnosed..$^{1-4}$ Magnetic resonance imaging (MRI) and magnetic resonance venography (MRV) have largely replaced computed tomography (CT) and catheter angiography in this context, and with respect to the superior sagittal sinus they provide images that are generally regarded as diagnostic and easy to interpret. ${ }^{5}$ Short of acute thrombosis, however, the lateral sinuses present a more difficult problem, with wide variations in radiological appearances inviting confusion between normal anatomical variants and disease. ${ }^{6}$ Nevertheless, these appearances are usually regarded as normal, even to the extent that the value of MRV in the investigation of patients with the typical clinical phenotype has been questioned. ${ }^{7}$

At variance with this is work from King et $a l^{8}$ and Karahalios et $a l^{9}$ who, using catheters passed into the intracranial venous sinuses through the jugular veins, have recorded raised pressures in the venous sinuses in the majority of patients with idiopathic intracranial hypertension. Sometimes these raised pressures seem to be secondary to raised central venous pressure, but more often they appear to be the result of focal stenotic lesions in the lateral sinuses obstructing cerebral venous outflow. There is a question over whether these lesions might be the cause or an effect of raised intracranial pressure. ${ }^{10-12}$ However, the symptomatic relief afforded some patients by dilatation and stenting of the lateral sinuses suggests that they may be causal in some cases. ${ }^{13-16}$

So what are the reasons for this disparity between the results of non-invasive investigation of venous sinuses in idiopathic intracranial hypertension (nearly always normal) and venography and manometry (nearly always abnormal)?
This study was carried out with a view to establishing whether there are characteristic appearances on MRV in idiopathic intracranial hypertension that are being routinely overlooked in clinical practice, and if so why.

\section{METHODS}

\section{Patient group}

All patients coded with a diagnosis of idiopathic intracranial hypertension between January 1998 and January 2001 at Addenbrooke's Hospital and the Norfolk and Norwich Hospital were identified. Their notes were retrieved by the audit departments at both hospitals and manually searched by two of us (BKO and $\mathrm{KH}$ ). Patients were diagnosed as having idiopathic intracranial hypertension if they had presented with a syndrome of raised intracranial pressure, usually manifest by headache and/or visual disturbance, without ventricular enlargement or an intracranial mass on imaging, with no evidence of venous sinus thrombosis, and with normal cerebrospinal fluid (CSF) constituents. ${ }^{17}$ Only patients who had undergone MRI and MRV as part of their diagnostic work up were included in the study.

Twenty two cases were identified. One patient whose diagnosis was subsequently changed to tension headache was excluded. Another, in whom the CSF pressure had never been measured, was also excluded. This left 20 patients who had had MRI and MRV, all of whom had been reported as showing no evidence of venous sinus disease (table 1). These scans were reviewed by two neuroradiologists together (JNPH and JHG) to look for evidence of acute thrombosis on the static images and abnormalities of flow on MRV. The presence of thrombosis was judged by standard diagnostic

Abbreviations: MRV, magnetic resonance venography 
Table 1 Demographic details of 20 patients with idiopathic intracranial hypertension

17 female, age 16 to 69 years (mean 30 )

3 male, age 17 to 57 years (mean 42 )

Body mass indices: $21-47 \mathrm{~kg} / \mathrm{m}^{2}$ (mean 35 )

Duration of symptoms before MRI: 1 week to 4 years (mean 5 months) CSF pressures $\left(\mathrm{cm} \mathrm{H}_{2} \mathrm{O}\right.$ ): 23 to 58 (mean 35)

criteria. ${ }^{18}$ The MRV appearances of the superior sagittal sinus and each lateral sinus were scored for each patient according to whether they were normal $(0)$, had one or more areas of focal narrowing ( 1 ), or contained one or more signal gaps (2). A superior sagittal sinus that was uniformly narrowed over its anterior third was regarded as normal. A lateral sinus that appeared uniformly narrow was also regarded as normal. Superior sagittal sinus scores were considered separately from lateral sinus scores. Lateral sinus scores were summated in each individual to give values from 0 (normal) to 4 (bilateral signal gaps) (fig 1).

\section{Control group}

Normal controls were recruited over a period of 18 months by poster advertisement or from the patient waiting list for noncerebral MRI and had none of the standard contraindications for MRI. All had MRI and MRV but first were screened by one of us (JNPH or JHG) using a questionnaire for symptoms relevant to their status as controls and categorised either as true (asymptomatic) normal or false (symptomatic) normal. Volunteers were informed before screening that their answers would not affect their eligibility for the study or a scan. Recruitment continued until there were 40 true normal subjects, matched for sex and for age within three years, with
Table 2 Demographic details of 40 asymptomatic normal volunteers

34 female, age 14 to 71 years (mean 30 ) 6 male, age 15 to 60 years (mean 42 )

the patient group (table 2). The MRIs and MRVs of this group were reviewed subsequently by two neuroradiologists (JNPH and JHG) and scored in the same way as the patient group.

\section{MRI and MRV}

All patients had had dual echo and Tl weighted MRI of the brain and either phase contrast or time of flight MRV. Inhouse scans were done on a GE 1.5 T Signa magnet using three dimensional phase contrast MRV, usually with velocity encoding of $15 \mathrm{~cm} / \mathrm{s}$ but occasionally using $30 \mathrm{~cm} / \mathrm{s}$ and once $40 \mathrm{~cm} / \mathrm{s}$. Separate acquisitions were made for the sagittal and lateral sinuses. Scans undertaken at the referring hospitals on a variety of machines were not repeated if they were of good quality.

All volunteers were scanned in-house on the same GE $1.5 \mathrm{~T}$ Signa magnet. All had sagittal T1 and axial T2 MRI. MRV was done using a three dimensional phase contrast technique with $15 \mathrm{~cm} / \mathrm{s}$ velocity encoding (time of repetition $25 \mathrm{~ms}$; flip angle $20^{\circ}$; time of echo $7.2 \mathrm{~ms}$ ). A sagittal slab of 12 contiguous slices, each $2.2 \mathrm{~mm}$ thick, was used to image the superior sagittal sinus and other midline venous structures. An axial slab of 28 slices, each $2.2 \mathrm{~mm}$ thick, was used to image the lateral sinuses. Scans were displayed as maximum intensity projection reconstructions for rating, with the source images available to resolve any uncertainty created by overlapping vessels.
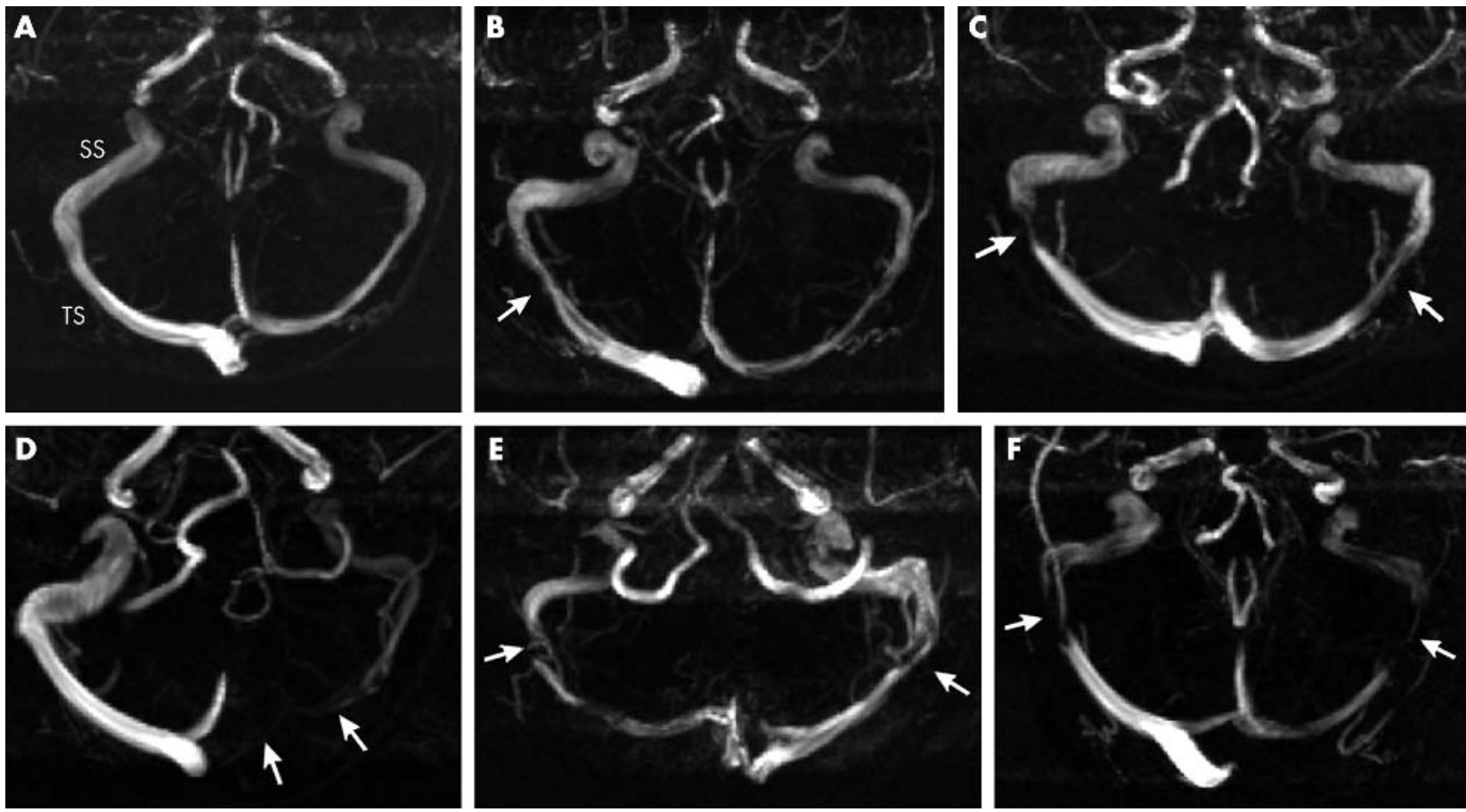

Figure 1 Lateral sinus scores and magnetic resonance venography (MRV) appearances. Three dimensional phase contrast MRV; axial views of lateral sinuses. Each lateral sinus scored either normal (0), with one or more focal narrowings (1), or with one or more signal gaps (2) and the results summated. (A) Score 0. (B) Score 1: focal narrowing right transverse sinus, arrow. (C) Score 2: focal narrowing both transverse sinuses, arrows. (D) Score 2: normal right transverse sinus, signal gap left transverse sinus, arrows. (E) Score 3: signal gap right transverse sinus-confirmed on source images, arrow; focal narrowing left transverse sinus, arrow. (F) Score 4: bilateral transverse sinus signal gaps, arrows. SS, right sigmoid sinus; TS, right transverse sinus. 


\section{RESULTS}

One hundred and forty volunteers were imaged, of whom 56 were designated as true normal; 46 of those could be matched with the patient group. The first 40 were recruited into the study. True normal controls only exceptionally complained of headache. Those excluded could not be age matched, or gave a history of headaches or other illness symptoms.

In the patient group there was no evidence of sinus thrombosis on the static images. On MRV the superior sagittal sinus showed uniform signal throughout its length in 18 cases and appeared relatively hypoplastic over its anterior third in two. The lateral sinuses showed a variety of appearances, the commonest being flow gaps present on both sides (score 4, fig $\mathrm{IF}$ ). Only one patient had strong uniform signal throughout both lateral sinuses (score 0, fig 1A). Figure 2 shows the frequency of the different appearances of the lateral sinuses in this group expressed in their scores as defined above.

In the control group static imaging was normal. On MRV the sagittal sinus showed an area of focal narrowing in five cases. There was some overlap with the patient group in the appearances of the lateral sinuses, but the distribution was significantly different $\left(\mathrm{p}<0.001\right.$ using the $\chi^{2}$ test for independence). The large majority of controls had strong uniform signal from at least one lateral sinus, and usually from both (score 0 or 1 ). In no control cases were flow gaps seen on both sides (fig 3 ).

\section{DISCUSSION}

In a patient with the appropriate clinical phenotype, the diagnosis of idiopathic intracranial hypertension is essentially one of exclusion of known causes of raised intracranial pressure. ${ }^{17}$ This has in many respects become easy with modern imaging. MRI will detect an intracranial mass or hydrocephalus. MRV and MRI will identify venous sinus thrombosis. ${ }^{5}$

Pertinent here-given that venous sinus thrombosis or cerebral venous outflow obstruction may be clinically indistinguishable from idiopathic intracranial hypertension-is the accuracy with which the radiological imaging excludes venous sinus disease. Once considered, acute thrombosis is not usually difficult to diagnose on the static images $^{18}$ and will be confirmed by abnormalities of flow on MRV. ${ }^{5}$ Chronic thrombosis, or partially recanalised thrombosis, is less obvious on the static $\mathrm{MRI}^{18}$ but may be recognisable on MRV. ${ }^{5}$ In this respect, MRV of the superior sagittal sinus does not normally present any problems in interpretation, a normal study generally giving uniform signal over the course of the sinus, thrombosis-new or old-usually producing quite extensive areas of reduced or absent signal. The lateral sinuses, however, are more difficult to assess, seeming to present a wide variation in the normal

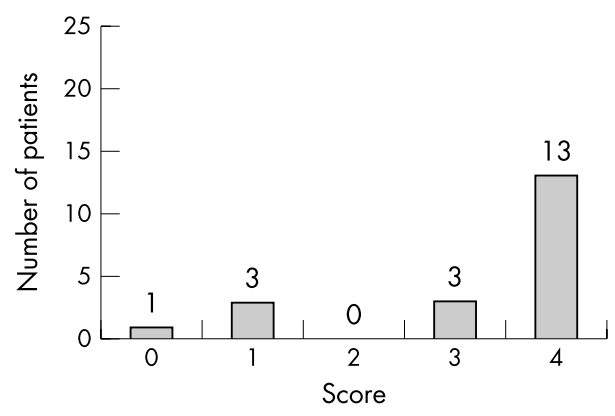

Figure 2 Lateral sinus scores in 20 patients with idiopathic intracranial hypertension.

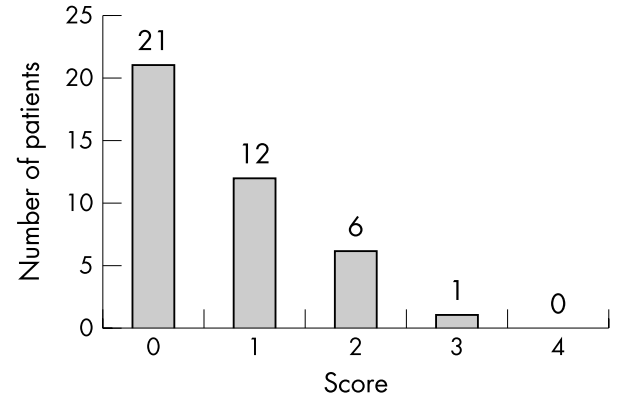

Figure 3 Lateral sinus scores in 40 normal asymptomatic controls matched for age and sex with the patient group.

anatomy. Either side may be dominant, either may be absent, and frequently they contain filling defects. ${ }^{6}{ }^{19}$ Ayanzen et al carried out MRV on 100 patients with normal MRI. ${ }^{6}$ They found defects in the lateral sinuses in $31 \%$, which they cautioned should not be mistaken for dural sinus thrombosis. Lee and Brazis, ${ }^{7}$ making allowances for similar appearances, found no evidence of sinus thrombosis in 20 patients with idiopathic intracranial hypertension.

These studies reflect current practice in the interpretation of MRV but make significant assumptions, the most important being that for the purpose of studying MRV a normal MRI qualifies a patient as a normal control. This is highly questionable. Ayanzen's patients were undiagnosed and their symptoms unspecified but it is probable-given that their MRI scans were normal-that many were being investigated for headache. ${ }^{20}$ Their conclusion ought therefore to have been that lateral sinus defects may be seen in $31 \%$ of patients with otherwise normal imaging, many of whom will probably have been referred for headache. Rao and Higgins examined the symptoms of 100 consecutive patients whose MRI scans were normal. ${ }^{21}$ They found that 31\% complained of headache and $22 \%$ of focal neurological symptoms. Seven per cent had acute seizures. These patients cannot be representative of the normal healthy population and particularly not of healthy venous sinuses. Headache, for example, is the cardinal symptom of venous sinus thrombosis and, moreover, has often been present for months before the diagnosis is made. 2223

There must therefore be a suspicion that our understanding of the normal appearances of the venous sinuses, based in part on clinical experience but mirrored in published reports, may be compromised by contamination of control groups with patients who have unrecognised venous sinus disease. Could this explain the failure of MRV to identify abnormalities of the venous sinuses in idiopathic intracranial hypertension?

To address this concern we selected a control group of "supernormals" from our volunteers. These subjects, whom we called true normal, only exceptionally ever suffered from headache and complained of no consistent symptoms that could conceivably be referred back to the head. Headache is a common symptom and inevitably there was a degree of subjectivity in assignment to one or other group. In the interests of the study objectives, however, admission to the true normal group was difficult, only one third of volunteers being considered sufficiently asymptomatic for inclusion in this category. Clearly, many truly healthy subjects must have been rejected from the true normal group (assuming that the symptoms they expressed were not indicative of pathology). These subjects would probably dilute any MRV abnormalities to be found in any analysis of the false normal group. However, their exclusion from the true normal group would have had no significant effect on the composition of this 
group, only on the time and effort required to attain the number of subjects needed for the study. On the other hand, also excluded from this group was a substantial proportion of volunteers who complained of quite significant symptoms, sometimes including severe and frequent headache. Some of these had attended for reassurance. Some had been investigated for the symptoms they described but given no specific diagnosis. One subject (who had previously had normal brain CT) had attended the ophthalmology clinic with a diagnosis of possible idiopathic intracranial hypertension.

The result of this selection process has been to expose clear differences between our two sample populations. We found that the great majority of patients with idiopathic intracranial hypertension had a distinctive pattern on MRV-signal gaps in both lateral sinuses - which was not seen in the control group. A smaller number had lesser defects but these were still unusual in the control group. Moreover, our control group showed substantially fewer defects in the lateral sinuses on MRV than recorded previously, ${ }^{6}$ lending credence to the concerns expressed above and suggesting that a reevaluation of normative data has become necessary.

What do these signal gaps mean? The signal in phase contrast MRV mainly comes from the bulk movement of protons (in blood) within a range of velocities chosen by the operator. ${ }^{24}$ As such, it is more a demonstration of flow than of anatomy, although some anatomical information is inevitably present in any study. A segment of signal void in a sinus implies that flow velocities over that segment are outside the range prescribed in the study. This does not necessarily mean thrombosis, and it may not necessarily be abnormalarachnoid granulations might cause a local alteration of blood flow around them, for example. However, it does raise the possibility of stenosis or occlusion.

In this context King et al and Karahalios et al, using more invasive techniques, ${ }^{89}$ have investigated the possibility that unrecognised venous sinus disease may be a frequent cause of idiopathic intracranial hypertension. With angiographic catheters passed into the intracranial venous sinuses through the jugular veins these workers found raised intrasinus pressures in many patients with idiopathic intracranial hypertension. Sometimes these high pressures appeared to be secondary to central venous hypertension but more often they seemed to be the result of stenotic lesions in the lateral sinuses obstructing cerebral venous outflow. This has led one group to propose intracranial venous hypertension as the final common pathway in the aetiology of idiopathic intracranial hypertension. ${ }^{9}$ Others, however, have since suggested that the sinus abnormalities observed in these cases are not the cause but the results of raised intracranial pressure (of unknown aetiology) inducing secondary sinus collapse. $^{10}$

Clearly, this latter issue is fundamental to questions regarding the aetiology of idiopathic intracranial hypertension and is not resolved by the current study. However, at least it is now possible to reconcile the non-invasive imaging with catheter venography and manometry. This means that, regardless of cause or effect, patients with idiopathic intracranial hypertension who have bilateral lateral sinus defects on MRV are likely to have raised venous pressures and stenotic lesions in their lateral sinuses. It means that, even in patients in whom frank venous sinus thrombosis has been excluded, MRV may still present an appearance that equates with raised intracranial pressure (an idea already explored by Quattrone et al with respect to chronic daily headache ${ }^{25}$ ). The possibility that this appearance may sometimes be indicating the cause of raised intracranial pressure is suggested by recent work documenting clinical improvement in cases of idiopathic intracranial hypertension following dilatation and stenting of the lateral sinuses. ${ }^{13-16}$ This pattern on MRV may thus in the future direct further investigation of these patients, and this concept should encourage a refinement of imaging techniques. ${ }^{26}$

Our patients were identified blind to a review of their MRI and MRV (although in the knowledge that they must have been reported as showing no evidence of sinus thrombosis). True normal controls were identified by interview before they were scanned. Otherwise this study was unblinded. Patients had had a variety of non-standardised MRI and MRV examinations. Controls were scanned according to a defined protocol. It would have been impossible to blind the observers to the imaging of patients versus controls. Inevitably, therefore, there will have been some bias in the reporting of the two groups, but the differences between them are so striking that this is unlikely to have influenced the results significantly.

\section{Conclusions}

MRV in patients with idiopathic intracranial hypertension commonly presents a pattern of bilateral lateral sinus defects rarely seen in asymptomatic controls. This result, at variance with published reports, implies a historical failure to separate patients with unrecognised venous sinus disease from healthy subjects in control populations, and implies a widespread failure to differentiate between normal variant and disease on MRV in clinical practice. This is highly relevant in the light of recent proposals regarding the aetiology and management of this condition.

\section{Authors' affiliations}

J N P Higgins, J H Gillard, Department of Radiology, Addenbrooke's Hospital, Cambridge, UK

B K Owlerm, Department of Neurosurgery, Royal Prince Alfred Hospital, Sydney, Australia

K Harkness, Department of Neurology, Addenbrooke's Hospital J D Pickard, Academic Neurosurgical Unit, Addenbrooke's Hospital Competing interests: none declared

\section{REFERENCES}

1 Ray BS, Dunbar HS. Thrombosis of the dural venous sinuses as a cause of "pseudotumor cerebri". Ann Surg 1951;134:376-86.

2 Janny $P$, Chazal J, Colnet $G$, et al. Benign intracranial hypertension and disorders of CSF absorption. Surg Neurol 1981;15:168-74

3 Medlock MD, Olivero WC, Hanigan WC, et al. Children with cerebral venous thrombosis diagnosed with magnetic resonance imaging and magnetic resonance angiography. Neurosurgery 1992;31:870-6.

4 Biousse V, Ameri A, Bousser MG. Isolated intracranial hypertension as the only sign of cerebral venous thrombosis. Neurology 1999;53:1537-42.

5 Vogl TJ, Bergman C, Villringer A, et al. Dural sinus thrombosis: value of venous MR angiography for diagnosis and follow up. Am J Radiol 1994; 162:1191-8

6 Ayanzen RH, Bird CR, Keller FJ, et al. Cerebral MR venography: normal anatomy and potential diagnostic pitfalls. Am J Neuroradiol 2000;21:74-8.

7 Lee AG, Brazis PW. Magnetic resonance venography in idiopathic pseudotumor cerebri. J Neuroophthalmol 2000;20:12-13.

8 King JO, Mitchell PJ, Thomson KR, et al. Cerebral venography and manometry in idiopathic intracranial hypertension. Neurology 1995;45:2224-8.

9 Karahalios DG, Rekate HL, Khayata MH, et al. Elevated intracranial venous pressure as a universal mechanism in pseudotumor cerebri of varying etiologies. Neurology 1996;46:198-202.

10 King JO, Mitchell PJ, Thomson KR, et al. Manometry combined with cervical puncture in idiopathic intracranial hypertension. Neurology 2002;58:26-30.

11 Corbett JJ, Digre K. Idiopathic intracranial hypertension; an answer to, "the chicken or the egg?" Neurology 2002;58:5-6.

12 Manometry combined with cervical puncture in idiopathic intracranial hypertension. NeurolLett 2002;59:963-4.

13 Higgins JNP, Owler BK, Cousins C, et al. Venous sinus stenting for refractory benign intracranial hypertension. Lancet 2002;359:228-30.

14 Owler BK, Allan R, Parker G, et al. Pseudotumour cerebri, CSF rhinorrhoea and the role of venous sinus stenting in treatment. $\mathrm{Br} J$ Neurosurg 2003;17:79-83.

15 Owler BK, Parker G, Halmagyi GM, et al. Pseudotumor cerebri syndrome: venous sinus obstruction and its treatment with stent placement. J Neurosurg 2003;98:1045-55.

16 Higgins JNP, Cousins C, Owler BK, et al. Idiopathic intracranial hypertension: 12 cases treated by venous sinus stenting. J Neurol Neurosurg Psychiatry 2003;74:1662-6. 
17 Sussman JD, Sarkies N, Pickard JD. Benign intracranial hypertension. Pseudotumour cerebri: idiopathic intracranial hypertension, Adv Tech Stand Neurosurg 1998;24:261-305.

18 Isensee C, Reul J, Thron A. Magnetic resonance imaging of thrombosed dural sinuses. Stroke 1994:25:29-34.

19 Huang YP, Okudera T, Ohta T, et al. Anatomic variations of the dural venous sinuses. In: Kapp JP, Schmidek HH, eds. The cerebral venous system and its disorders. Orlando: Grune and Stratton, 1984:109-68.

20 Reid AC, Matheson MS, Teasdale G. Volume of the ventricles in benign intracranial hypertension. Lancet 1980;i:7-8.

21 Rao S, Higgins JNP. What is a normal magnetic resonance venogram? [Abstract] Meeting of the British Society of Neuroradiologists, Winchester, 2002 October 11
22 Kalbag RM. Cerebral venous thrombosis. In: Kapp JP, Schmidek HH, eds. The cerebral venous system and its disorders. Orlando: Grune and Stratton, 1984:505-36.

23 Bousser MG, Chirs JC, Bories J, et al. Cerebral venous thrombosis - a review of 38 cases. Stroke 1985;16:199-213.

24 Westbrook C, Kaut C, eds. Vascular and cardiac imaging. In: MRI in practice. Oxford: Blackwell Scientific Publications, 1993:175-203.

25 Quattrone A, Bono F, Oliveri RL, et al. Cerebral venous thrombosis and isolated intracranial hypertension without papilloedema in $\mathrm{CDH}$. Neurology 2001;57:31-6.

26 Farb RI, Vanek I, Scott JN, et al. Idiopathic intracranial hypertension: the prevalence and morphology of sinovenous stenosis. Neurology 2003:60:1418-24.

\section{NEURONLINE}

\section{Neurosciences on the internet: www.neuroguide.com}

$\mathrm{N}$ eurosciences on the internet is a large and well established database of URLs, titles, and keywords of resources available on the internet that are likely to be of interest not only to neuroscientists but also to clinicians, patients, and their carers. Created and maintained by Neil Busis, a Pittsburgh neurologist, the database is now 10 years old and continues to be updated on a regular basis. The indexed resources are grouped under several arbitrary but clear titles to allow for browsing, and can also be accessed by a basic but functional search engine. The graphics and navigation around the site are basic but effective, but the real interest of the site lies in the assembled content. One of the advantages of indices compiled by humans (rather than automatically assembled) is that the resources indexed can be screened for quality and organised by relevance. This works very effectively in some areas of neurosciences on the internet. For example, the section on human neurological diseases provides a very effective way to directly find other websites and sources of information on specific disease entities. In other areas, the reliance on a human compiled index based on submitted information is less successful. For example, the documentation of the websites of academic institutes is only partial and limited, especially outside North America, and it might be quicker in this case to rely on an automated search engine such as Google. For those not directly searching a specific source of information, the site also offers the facility to play 'neuroroulette', automatically selecting a link from the database at random. My twist of the neuroroulette wheel led to the little

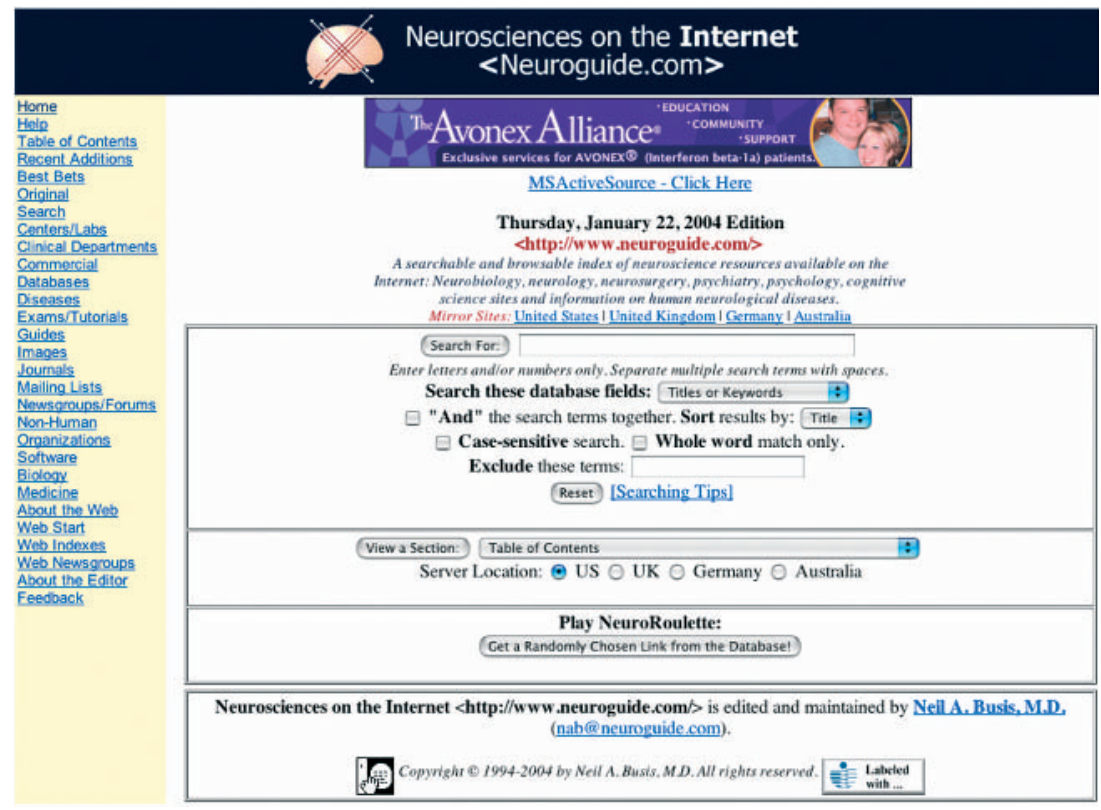

known (to me, at least) website, The Lurkers guide to stomatopods (http://www.blueboard. com/mantis/). Although perhaps not directly relevant to clinical practice, it was undeniably interesting to read about predatory crustaceans and their visual system as a result of visiting Neuroscience on the internet! Neurosciences on the internet is probably the largest indexed database of clinical and basic neuroscience related URLs, and therefore a great resource in its own right for the clinician or scientist looking for specific sources of information.

G Rees Institute of Cognitive Neuroscience, University College London, 17 Queen Square, London WCIN 3AR, UK; g.rees@fil.ion.ucl.ac.uk 